\title{
SPARC ectopic overexpression inhibits growth and promotes programmed cell death in acute myeloid leukemia transformed from myelodysplastic syndrome cells, alone and in combination with Ara-C treatment
}

\author{
QING NIAN $^{1 *}$, JIANXIANG CHI $^{2 *}$, QING XIAO $^{1}$, CHUNMEI WEI $^{1}$, PAUL COSTEAS $^{2}$, \\ ZESONG YANG ${ }^{1}$, LIN LIU ${ }^{1}$ and LI WANG ${ }^{1}$ \\ ${ }^{1}$ Department of Hematology, The First Affiliated Hospital of Chongqing Medical University, Chongqing 400016, P.R. China; \\ ${ }^{2}$ The Center for the Study of Haematological Malignancies, Nicosia 2032, Cyprus
}

Received March 9, 2015; Accepted May 12, 2015

DOI: 10.3892/or.2015.4114

\begin{abstract}
Secreted protein acidic and rich in cysteine (SPARC) has a complex and pleiotropic biological role in cell life during disease. The role of SPARC in myelodysplastic syndrome (MDS) is not yet fully understood. In the present study, we investigated the role of SPARC protein overproduction in the proliferation and apoptosis of SKM-1 cells, an acute myeloid leukemia cell line transformed from MDS. SKM-1 cells were infected with the pGC-GV-SPARC vector. The cells were then assessed for proliferation and cell death following treatment with low-dose cytosine arabinoside (Ara-C). The microarray analysis results revealed that samples from SPARC-overexpressed cells compared to SPARC protein, in SKM-1 cells led to proliferation inhibition and promoted programmed cell death and these effects were greater when treated with Ara-C. The mRNA and protein expression levels of SPARC were detected by SPARC overexpression in cells treated with Ara-C resulting in a significant upregulation of the mixed lineage kinase domain-like $(M L K L)$ gene expression and five other genes. The results showed that the necrotic signaling pathway may play a role when the two conditions were combined via the upregulation of the MLKL protein. MLKL upregulation in SPARC overexpressed cells treated with Ara-C, indicates necrosis as a possible cell death process for the SKM-1 cells under these stringent conditions.
\end{abstract}

Correspondence to: Dr Li Wang, Department of Hematology, The First Affiliated Hospital of Chongqing Medical University, 1 Youyi Road, Yuzhong District, Chongqing 400016, P.R. China

E-mail: erin_wang77@hotmail.com

${ }^{*}$ Contributed equally

Key words: SPARC gene, myelodysplastic syndrome, transfection, Ara-C

\section{Introduction}

Myelodysplastic syndrome (MDS) is a diverse group of neoplastic bone marrow disorders, characterized by blood cytopenia, dysplasia in the cell morphology of bone marrow, and blood defects in the normal differentiation and proliferation of hematopoietic precursors (1). MDS is a preleukemic pathological condition with a risk of transformation to acute myeloid leukemia of 30-40\% (1-3). Chromosomal abnormalities are observed in 50-60\% of patients with MDS, with the most frequent single cytogenetic abnormalities including $\operatorname{del}(5 q)$, monosomy 7 or $\operatorname{del}(7 q)$, trisomy 8 and $\operatorname{del}(20 q)$ (2).

One of the best characterized MDS-associated alterations is the $\operatorname{del}(5 q)$ that occurs either as a stand-alone chromosomal alteration or in combination with other chromosomal abnormalities presenting a more complex phenotype $(4,5)$. The $\operatorname{del}(5 q)$ is found with a frequency of $10-15 \%$ in MDS patients and is a marker for a favorable outcome when this occurs as an isolated cytogenetic aberration (5-7), although it is associated with a poor prognosis in high-risk MDS and de novo AML (8).

A common deleted region (CDR) has been identified covering the area of 5q31-32, where two alternative missing segments have been reported, one proximal deleted entity associated with advanced MDS conditions and a second distal deleted region associated with the $5 \mathrm{q}$-deletion syndrome with better prognosis (9). Loss of the CDR region ablates 44 genes and results in haploinsufficiency conditions for many of these gene products, where the levels of gene products are not sufficient for the physiological cell function. One of the genes in this area is the osteonectin (SPARC) gene, known to code for a secreted, counter-adhesive, matricellular protein (cysteinerich protein) with a diversity of biological functions associated with morphogenesis, remodeling, cell migration and proliferation (10). The expression of the SPARC gene has been found to be variable in different cancer types. This expression is evidently high in breast and colorectal cancer $(11,12)$, but low in prostate and lung cancer $(13,14)$. Moreover, SPARC gene expression has been associated with the regulation of cell biology, at the level of proliferation and apoptosis, as well 
as with responses to immunoregulatory treating agents in a complex and not well-defined manner $(15,16)$. In MDS, patients with a stand-alone $\operatorname{del}(5 \mathrm{q})$ treated with lenalidomide, markedly upregulated SPARC gene expression during and after the treatment, resulting in proliferation inhibition and apoptosis of the cancer cells (16). A beneficial effect has also been shown in CML patients where SPARC was increased during and for a long period after treatment with imatinib, which was shown to synergize with the drug to inhibit the growth of K562 cells (17). By contrast, SPARC overexpression in $\mathrm{CN}-\mathrm{AML}$ has been recently associated with adverse outcomes in patients and aggressive leukemia growth in murine models of AML (18). In addition, haploinsufficiency of SPARC in the $5 q$-syndrome may increase the adhesion of the hematopoietic stem cells to the supporting stromal cells and provide a clonal advantage (19).

Cytarabine [cytosine arabinoside, (Ara-C)] is commonly used as a cytotoxic agent for the chemotherapeutic treatment of MDS. It is a pyrimidine antimetabolite that interferes with the DNA synthesis of cells in S-phase of the cell cycle via inhibition of DNA polymerase. In the clinic, it has been found that Ara-C cell apoptosis is dose-dependent and may play a role in cell cycle regulation by induction of quiescence in proliferating tumor cells (20). It is used in low doses and often in combination with other therapeutic drugs, especially in patients with intermediate or high IPSS scores, who cannot tolerate high intensity chemotherapy or bone marrow transplantation (2). It is also a viable option for treating patients with complex cytogenetic phenotype (21). In the present study, we investigated the effects on cell proliferation and death responses of MDS/AML SKM-1 cells under two conditions: i) the SPARC gene overexpression and the resulting increase of its protein levels, and ii) their treatment with low levels of Ara-C, alone or in combination. We demonstrated that SPARC gene overexpression and increased protein levels resulted in inhibition of proliferation in SKM-1 cells and a concomitant increase of programmed cell death. Furthermore, we provide evidence that the SKM-1 cells may undergo cell death through an alternative to the classical apoptosis/necrosis pathways, that of necroptosis, showing overexpression of the major pathway protein, mixed lineage kinase domain-like (MLKL).

\section{Materials and methods}

Construction of recombinant SPARC-expressing lentivirus vector. The construction of a recombinant lentivirus vector-expressing SPARC gene has been described in a previous study (22). Briefly, SPARC cDNA was amplified by means of reverse transcription polymerase chain reaction (RT-PCR), using SPARC sequence-specific primers (Table I) and was subcloned into the lentiviral vector pGC-GV to develop a recombinant lentiviral construct designated as pGC-GV-SPARC (Jikai Biomedical Corp., Shanghai, China). The vector intrinsically carries the gene for GFP for detecting expression efficiency. According to the operation manual, packaging of the retrovirus in 293 cells, Lipofectamine 2000, pHelper 1.0 and pHelper 2.0 (Jikai Biomedical Corp.) were used. Supernatants were then collected for the determination of viral titers. A control empty lentivirus vector carrying the GFP gene NC-GFP-LV was used to infect SKM-1 cells.
Table I. qPCR primers used in this study.

\begin{tabular}{llc}
\hline \multirow{2}{*}{ Genes } & \multicolumn{1}{c}{ Primers } & $\begin{array}{c}\text { Product } \\
\text { length } \\
\text { (bp) }\end{array}$ \\
\hline SPARC & F: 5'-GGCCTGGATCTTTCTCCTT-3' & 126 \\
& R: 5'-CCCACAGATACCTCACCTC-3' & \\
3-actin & F: 5'-CCACGAAACTACCTTCAACTAA-3' & 132 \\
& R: 5'-GTGATCTCCTTCTGCATCCTGT-3' & \\
CD164L2 & F: 5'-CACCCTCACCTCCAAGGAC-3' & 106 \\
& R: 5'-GTGACCTTGAGTTCCCAGA-3' & \\
MLKL & F: 5'-TGTCTTTTCTCTCGTAGTT-3' & 184 \\
& R:5'-GAAGTCTGTGTTTCCTCA-3' & \\
GALR3 & F: 5'-CCCCTCGCAAGCAGCCTCTGGG-3' & 118 \\
& R: 5'-TGCAGGGCGTGCTTGAGGGG-3' & \\
ELP5 & F: 5'-ATCTGGACCCTCCTACCTCTGG-3' & 157 \\
& R: 5'-GATGCAGGCCTTCCAAGTTCT-3' & \\
NT5C2 & F: 5'-TGTTCTGAAAGCTGGGAGCA-3' & 136 \\
& R: 5'-AGAAACTGACCTGAGTTTAA-3' & \\
AFM & F: 5'-TTCATTTTTATTTTTATAG-3' & 141 \\
& R: 5'-AAGTTGCCAGAAGGAACC-3' & \\
& &
\end{tabular}

$\mathrm{F}$, forward; R, reverse; SPARC, secreted protein acidic and rich in cysteine; CD164L2, CD164 sialomucin-like-2; MLKL, mixed lineage kinase domain-like; GALR3, galanin receptor 3; ELP5, elongator acetyltransferase complex subunit 5; NT5C2, 5'-nucleotidase cytosolic II; AFM, afamin.

Cell culture and infection protocol. The MDS/AML cell line SKM-1 was kindly provided by Professor Zhou at the Tongji Medical College, Huazhong University of Science Technology and cultured in RPMI-1640 medium supplemented with $10 \%$ fetal bovine serum (Gibco-BRL, Grand Island, NY, USA) in a humid atmosphere at $37^{\circ} \mathrm{C}$ with $5 \% \mathrm{CO}_{2}$. For the infection protocol, the cells were cultured in 6 -well plates $\left(10^{6} \mathrm{cells} / \mathrm{ml} /\right.$ well), and infected with the lentivirus vectors at a multiplicity of infection (MOI) of 100 for $10 \mathrm{~h}$. The cells were then washed and basic medium free of infecting particles was added. After 4 days, the cells were assessed under a fluorescence microscope and flow cytometry for the evaluation of transduction efficiency of the vector was used measurement of GFP expression.

Quantitative-PCR. Total RNA from each test group was extracted from cells using RNAiso Plus (Takara Biotechnology, Dalian, China) and used for cDNA synthesis. Each reaction contained $12.5 \mu 1$ Taq (Takara Biotechnology), $1 \mu 1$ of each primer $(10 \mu \mathrm{mol} / \mathrm{l}), 2 \mu \mathrm{l} \mathrm{cDNA}$ template $(50 \mathrm{ng} / \mu \mathrm{l})$ and $8.5 \mu \mathrm{l}$ $\mathrm{ddH}_{2} \mathrm{O}$. PCR primers are listed in Table I. The cycling parameters used were: $97^{\circ} \mathrm{C}$ for $5 \mathrm{~min}, 30$ cycles at $97^{\circ} \mathrm{C}$ for $1 \mathrm{~min}$, $56^{\circ} \mathrm{C}$ for $30 \mathrm{sec}, 72^{\circ} \mathrm{C}$ for $30 \mathrm{sec}$, and a final extension at $72^{\circ} \mathrm{C}$ for 7 min. Quantitative qPCR was performed using an ABI PRISM 7500 Real-Time PCR system (Applied Biosystems, Foster City, CA, USA). The total reaction composition was $25 \mu \mathrm{l}$ : SYBR Premix EX Taq II $(12.5 \mu \mathrm{l}), 1 \mu \mathrm{l}$ of each primer (10 $\mu \mathrm{mol} / \mathrm{l}), 2 \mu \mathrm{l} \mathrm{cDNA}$ template $(50 \mathrm{ng} / \mu \mathrm{l})$, and $8.5 \mu \mathrm{l}$ of $\mathrm{ddH}_{2} \mathrm{O}$. RT-PCR results were analyzed using Quantity One software (Bio-Rad, Hercules, CA, USA). The primers were 
designed using Primer 5 software and synthesized by Takara Biotechnology (Table I).

Cell proliferation assay. Cell proliferation was determined using the MTS assay. The cells were seeded in $5 \times 10^{2}$ cells/well in 96-well plates. The cells were divided into six groups: i) pGC-GV-SPARC-infected cells + Ara-C; ii) pGC-GV-SPARC-infected cells; iii) NC-GFP-LV-infected cells + Ara-C; iv) SKM-1 cells + Ara-C; v) NC-GFP-LV-infected cells; and vi) SKM-1 cells. The lentivirus vector pGC-GVSPARC was used to overexpress SPARC in the infected SKM-1 cells and NC-GFP-LV served as the empty vector for the negative control. For the MTS proliferation assay, $20 \mu 1$ of MTS (Promega, Madison, WI, USA) was added to each well and incubated at $37^{\circ} \mathrm{C}, 95 \%$ humidity and $5 \% \mathrm{CO}_{2}$ for $1 \mathrm{~h}$. The optical density at $490 \mathrm{~nm}$ was read with an enzyme immunoassay instrument (Bio-Rad). Cell growth inhibition rate (\%) was defined and calculated according to the formula: [1 - experimental group D (490)/control group D (490)] x 100. The experiments were performed in triplicate and an average value was obtained.

Annexin $V$ and 7-AAD apoptotic assay. The cells were collected $\left(10^{6}\right.$ cells $\left./ \mathrm{ml}\right)$ and washed twice with PBS, suspended in $200 \mu \mathrm{l}$ binding buffer, $1 \mu \mathrm{l}$ Annexin V-PE and $5 \mu 1$ 7-AAD (KeyGen Biotech, Shanghai, China) for $15 \mathrm{~min}$ in the dark. The infection efficiency and cell death were determined by flow cytometry using the CellQuest software (BD Biosciences).

Western blot analysis. The cells were lysed in $100 \mu \mathrm{l}$ RIPA buffer supplemented with $1 \mu \mathrm{l} \mathrm{PMSF}$, and protein concentration of the lysate was determined using a BCA Protein Assay kit (Beyotime, Beijing, China). A total of $50 \mu \mathrm{g}$ of protein per lane was separated by SDS-PAGE and transferred to PVDF membranes. The membranes were blocked with $5 \%$ skimmed milk for $2 \mathrm{~h}$, then incubated overnight at $4^{\circ} \mathrm{C}$ with primer antibodies (mouse anti-human monoclonal antibody, dilution, 1:1,000; Abcam, Cambridge, UK) for SPARC and $\beta$-actin followed by incubation with HRP-conjugated goat anti-rabbit (dilution, 1:1,000; ImmunoWay, Newark, DE, USA) for $1 \mathrm{~h}$ at $37^{\circ} \mathrm{C}$. The membranes were washed four times with TBST and developed using the ECL (Beyotime) method. Band intensity was analyzed using Quantity One software (Bio-Rad).

Affymetrix experiments and microarray data analysis. Total RNA was extracted from the cells using TRIzol (Takara Biotechnology) and according to the manufacturer's instructions. The RNA samples examined were obtained from SKM-1 cells, SKM-1 cells treated with Ara-C, and pGC-GV-SPARC-infected cells treated with Ara-C. For each sample, $50 \mathrm{ng}$ of total RNA were amplified and labeled with the Two-Cycle cDNA Synthesis and the Two-Cycle Target Labeling and Control reagent packages (both from Affymetrix, Santa Clara, CA, USA) according to the manufacturer's instructions. The cell intensity calculation and scaling were then performed using GeneChip Operating software (GCOS). Data analysis was performed using GeneSpring 7.3 (Agilent Technologies, Santa Clara, CA, USA) and the clustering analysis.
A

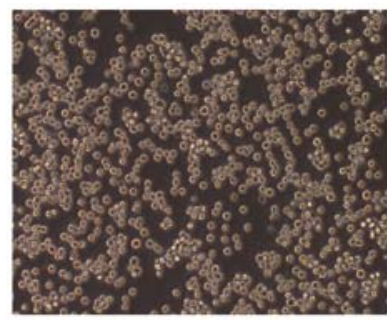

B

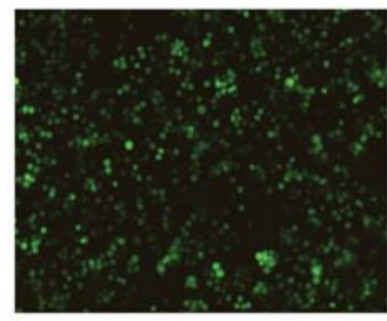

$\mathrm{C}$

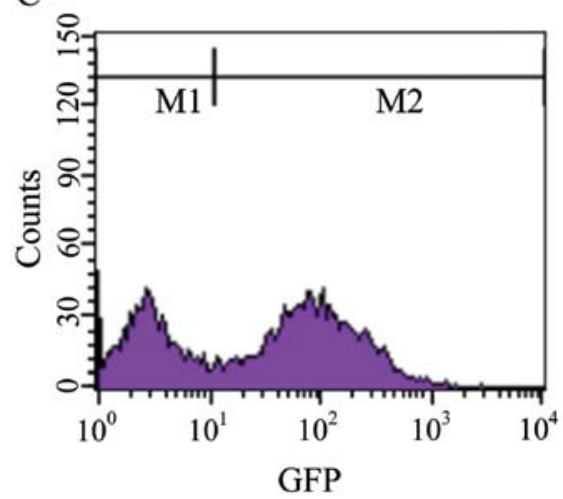

Figure 1. Infection of SKM-1 cells by SPARC-expressing vector (pGC-GVSPARC) results in stable transduction and ectopic expression of SPARC. (A) Normal SKM-1 cells; and (B) SKM-1 cells infected with pGC-GV-SPARC. (C) The transduction efficiency detected by flow cytometry. M1, no EGFP; M2, EGFP. The transduction efficiency was $68 \%$. SPARC, secreted protein acidic and rich in cysteine.

Statistical analysis. All results were expressed as the mean $\overline{\mathrm{X}} \pm$ SE and analyzed by GraphPad Prism 5 software and each experiment was repeated three times. The comparison among the groups was analyzed by one-way ANOVA. P-value $<0.05$ was considered statistically significant.

\section{Results}

Lentivirus-mediated SPARC overexpression by SKM-1 cells. SKM-1 cells express a relatively high endogenous level of SPARC mRNA (22). The expression of SPARC protein was multiplied ectopically in SKM-1 cells, to create a suitable in vitro model for the SPARC-overexpression experiments. SKM-1 cells co-cultured with the pGC-GV-SPARC lentivirus vector and the empty negative control vector (NC-GFP-LV), resulted in exhibition of the expression of GFP from the cells, $96 \mathrm{~h}$ after infection, demonstrating stable ectopic gene expression (Fig. 1A and B). The efficiency of vector transduction was $>67 \%$, calculated from the flow cytometry results for GFP detection (Fig. 1C). The qPCR of mRNA samples isolated from pGC-GV-SPARC infected SKM-1 cells, detected an increased number of SPARC transcripts of a magnitude of 15 times, when compared to normal SKM-1 and NC-GFP-LV-infected SKM-1 cells. The data were normalized to $\beta$-actin gene 

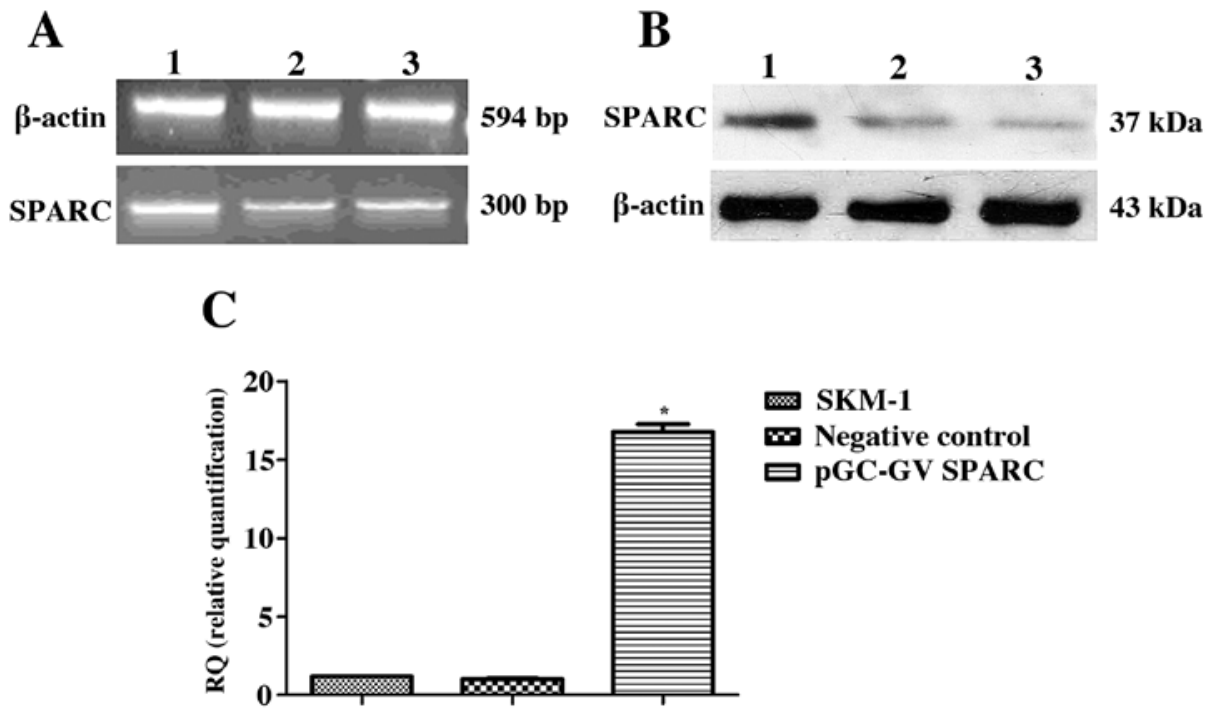

Figure 2.NormalSPARC expression and overexpression in SKM-1 cells.(A)RT-PCR showing the expression of SPARCmRNA.Lanes 1,pGC-GV-SPARC-infected SKM-1 cells, 2, SKM-1 cells infected with empty vector control and 3, normal SKM cells. (B) Protein was measured by western blot analysis showing the expression of SPARC protein. Lanes 1, pGC-GV-SPARC-infected SKM-1 cells, 2, SKM-1 cells infected with empty vector control and 3, normal SKM-1 cells. (C) Quantitative PCR data showing the relative expression of SPARC mRNA in SKM-1 cells in different conditions, ${ }^{*}<<0.05$. SPARC, secreted protein acidic and rich in cysteine; RT-PCR, reverse transcription polymerase chain reaction.

Table II. Effects of low-dose Ara-C inhibit SKM-1 cells detected by MTS (mean \pm SD) $\%$.

\begin{tabular}{lccr}
\hline Groups & $24 \mathrm{~h}$ & $48 \mathrm{~h}$ & $72 \mathrm{~h}$ \\
\hline SPARC overexpress + Ara-C & $44.451 \pm 1.545$ & $62.152 \pm 1.081$ & $76.417 \pm 1.372$ \\
SPARC overexpress & $16.111 \pm 1.041$ & $21.311 \pm 1.402$ & $30.524 \pm 1.056$ \\
Negative control + Ara-C & $24.877 \pm 2.742$ & $36.573 \pm 1.292$ & $43.892 \pm 1.223$ \\
Negative control & $4.483 \pm 1.491$ & $6.996 \pm 1.577$ & $7.112 \pm 2.342$ \\
SKM-1 + Ara-C & $23.831 \pm 1.357$ & $31.197 \pm 1.231$ & $41.889 \pm 1.352$ \\
SKM-1 & - & - & - \\
\hline
\end{tabular}

Ara-C, cytosine arabinoside; SPARC, secreted protein acidic and rich in cysteine.

expression (Fig. 2A). This difference of increased SPARC gene expression in pGC-GV-SPARC-infected SKM-1 cells, reached high statistical significance $(\mathrm{P}<0.01)$ when compared to the normal SKM-1 cells and to cells expressing NC-GFPLV (Fig. 2C). Although SPARC protein is secreted, western blot analysis on SKM-1 cells infected with pGC-GV-SPARC, confirmed overproduction of the SPARC protein. Protein extracts from the pGC-GV-SPARC cells had significantly higher SPARC proteins compared to the negative vector control cell line and the normal SKM-1 cells (Fig. 2B).

SPARC overexpression from SKM-1 cells and SPARC overexpression combined with Ara-C treatment, inhibits SKM-1 cell proliferation. The proliferation rate of the SKM-1 cells when they ectopically overexpress SPARC protein and following administration of low-dose Ara-C was assessed at three different time-points. Cells that were stably transduced with pGC-GV-SPARC were tested for their proliferation efficiency and were compared with the same cells after treatment with a low dose of Ara-C and normal SKM-1 cells. Measurements were taken at 24, 48 and $72 \mathrm{~h}$ after treatment with Ara-C. A control group, SKM-1 cells after infection with the empty lentivirus vector was also assessed. A small proliferation inhibition was exerted on SKM-1 cells when infected with the empty lentiviral control vector (negative control). This inhibition was increased from $4.5 \%$ of the normal SKM-1 cells proliferation rate at $24 \mathrm{~h}$, to $7 \%$ after $72 \mathrm{~h}$ (Table II). Treatment of the same negative control cells with Ara-C resulted in a marked increase on proliferation inhibition to $25 \%$ at $24 \mathrm{~h}$ and to $44 \%$ at $72 \mathrm{~h}$ post-treatment. Similar results were obtained when normal SKM-1 cells were infected with Ara-C (Table II). Notably, SPARC-overexpressing SKM-1 cells demonstrated a reduced proliferation rate of $16 \%$ of the normal cell proliferation rate at $24 \mathrm{~h}$ and $31 \%$ at $72 \mathrm{~h}$ without any treatment. The inhibitory effect of SPARC protein overexpression, closely resembled the one achieved by Ara-C treatment of normal SKM-1 cells. Moreover, SPARC-overexpressing cells when treated with low-dose Ara-C demonstrated an additive inhibitory effect, starting from $45 \%$ of the normal proliferation rate at $24 \mathrm{~h}$ and progressing to $62 \%$ at $48 \mathrm{~h}$ and reaching $76 \%$ at $72 \mathrm{~h}$ post-treatment. These results were statistically significant when compared to the negative control values $(\mathrm{P}=0.014)$. 
A

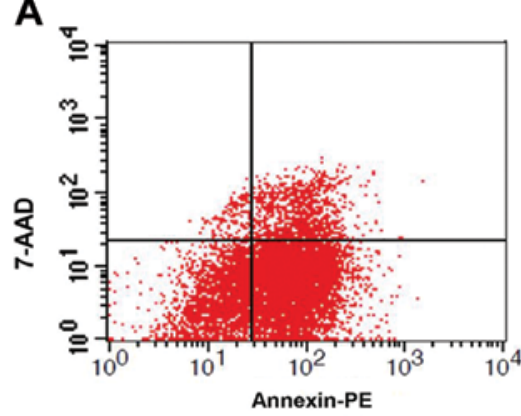

C

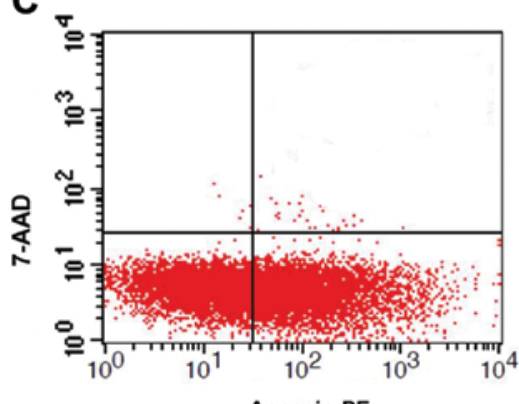

E

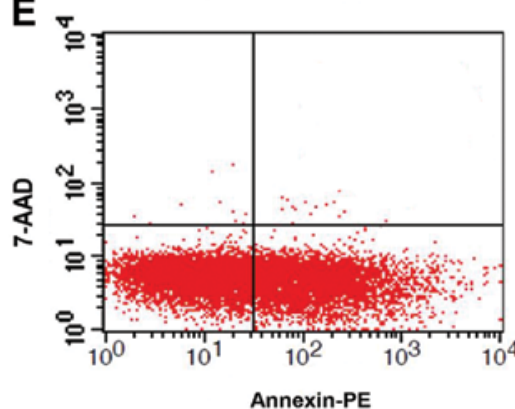

Annexin-PE

\section{G}

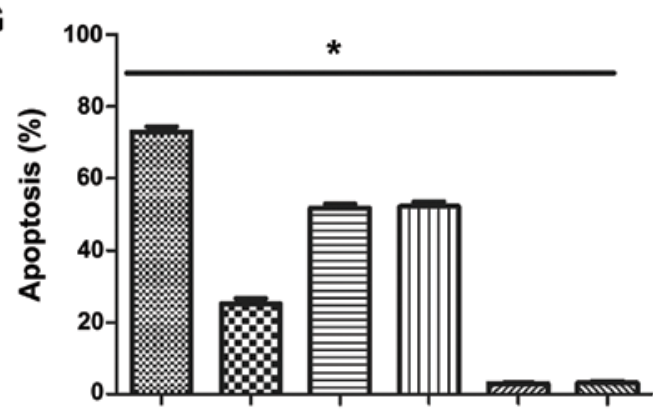

B

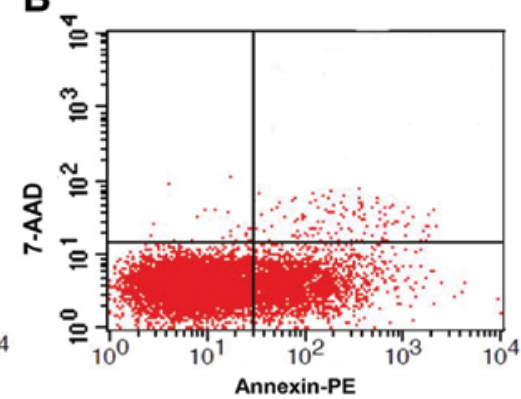

D

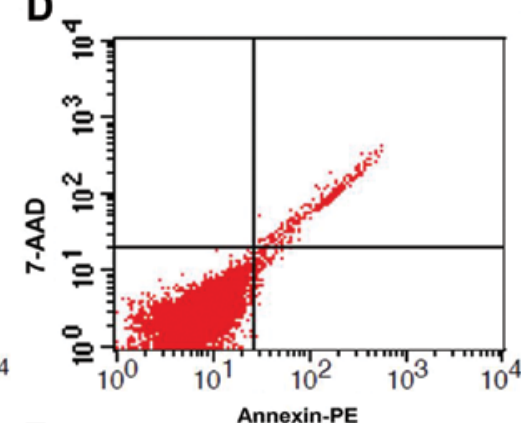

$\mathbf{F}$

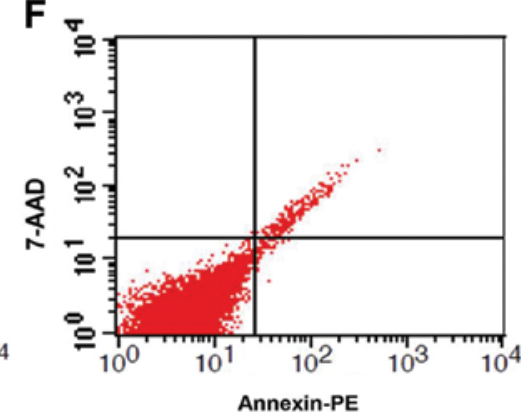

pGC-GV SPARC+Ara-C

W PGC-GV SPARC

Degative control+Ara-C

س.

(Im) Negative control

बलI SKM-1

Figure 3. Apoptotic effects on SKM-1 cells after SPARC overexpression and Ara-C treatment. All assays used SKM-1 cells. (A) pGC-GV-SPARC-infected and Ara-C treated and (B) pGC-GV-SPARC-infected cells. (C) Empty vector negative control infection and Ara-C treatment and (D) empty vector negative control infection. (E) Normal SKM-1 cells treated with Ara-C and (F) normal SKM-1 cells. (G) Apoptosis results show significan difference ("P<0.01) vs. other groups. Data were analyzed using GraphPad Prism 5 software. SPARC, secreted protein acidic and rich in cysteine; Ara-C, cytosine arabinoside.

SPARC overexpression and Ara-C induce cell apoptosis in SKM-1 cells. Having shown that SPARC overexpression results in proliferation inhibition, and that treatment with Ara-Cresulted in an marked increase of this inhibitory effect, we investigated whether there is a corresponding apoptotic effect on the cells. Flow cytometric analysis for apoptosis of the SKM-1 cells and SKM-1 cells infected with empty vector control, showed no measurable apoptotic effect between them. By contrast, a 16\% increase in apoptosis of pGC-GV-SPARC-infected SKM-1 cells was measured compared to the control groups (Fig. 3). The increase in apoptosis cannot be attributed to the infecting vector, as the empty negative control vector did not measurably elevate the number of apoptotic cells infected. It is evident therefore, that overexpression of SPARC protein in pGC-GV-SPARC-infected SKM-1 cells exerts an apoptotic effect on the cells. Having established an apoptotic relationship to the increased levels of SPARC, we determined whether overexpression of SPARC may influence the treatment effect of the pGC-GV-SPARC-transduced SKM-1 cells to Ara-C. We performed a flow cytometric analysis to evaluate cell death responses on SPARC-overexpressing SKM-1 cells after treatment with Ara-C. We found that $>70 \%$ of cells die $24 \mathrm{~h}$ after treatment, which is a $>20 \%$ increase of death in normal SKM-1 cell numbers treated with Ara-C $(\mathrm{P}<0.05)$. 


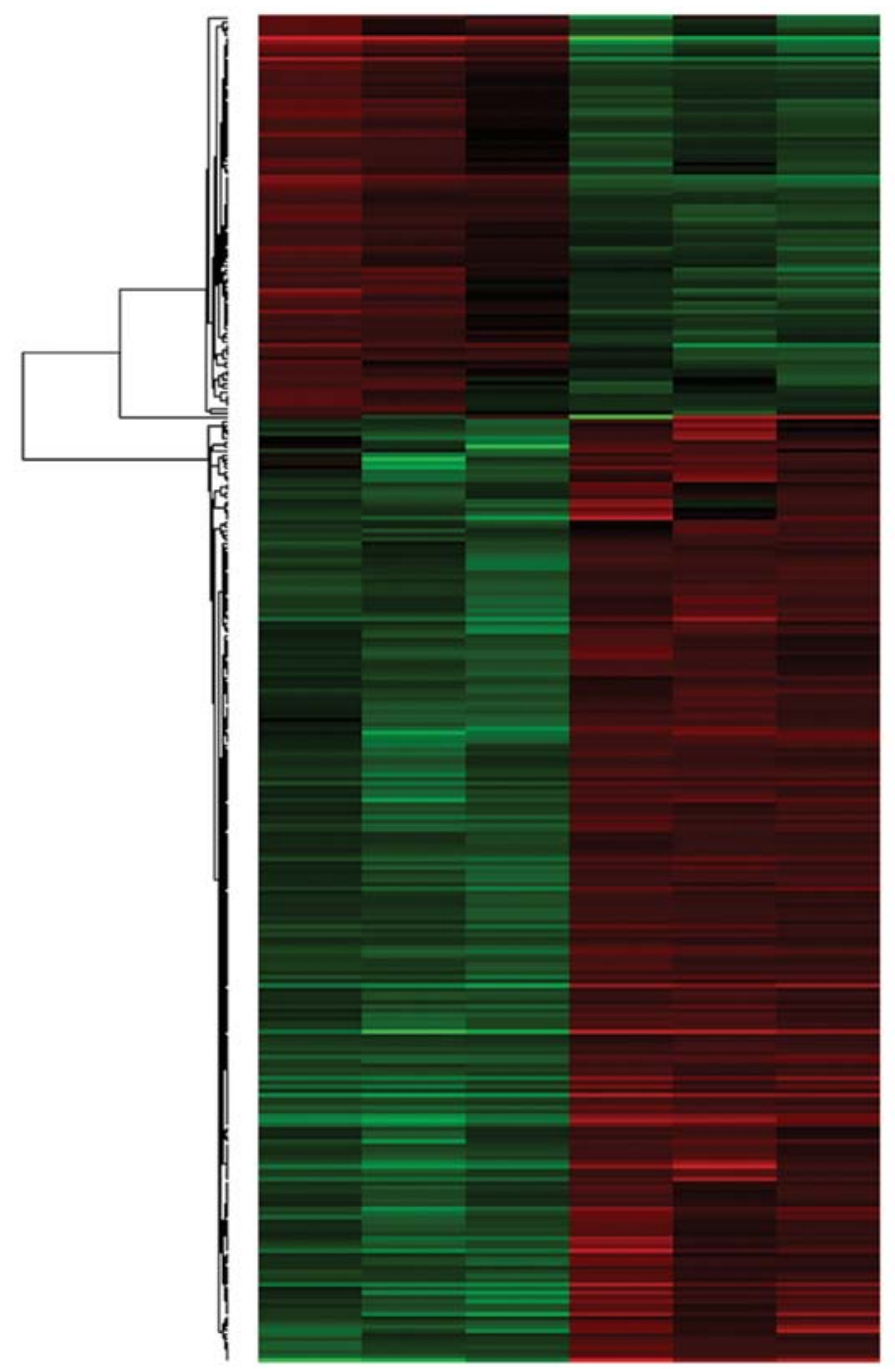

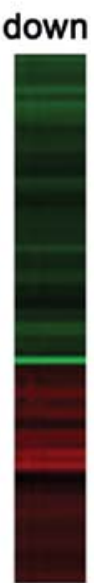

up

Figure 4. Hierarchical clustering of 672 differentially expressed genes in the SKM-1 cells treated with Ara-C and SPARC-overexpressing SKM-1 cells treated with Ara-C. Each row shows a single affymetrix probe set. SPARC, secreted protein acidic and rich in cysteine; Ara-C, cytosine arabinoside.

Gene transcription responses to Ara-C exposure. Our experiment was based on a comparison of two study groups: SKM-1 cells treated with Ara-C and SPARC-overexpressing SKM-1 cells treated with Ara-C. Analysis of the microarray results showed the upregulation of expression in 566 genes and downregulation in 106 gene transcripts levels (Fig. 4). The three genes with a high upregulation and the three downregulated genes, according to their relevance to cell death and disease were selected for further investigation. These genes were: $M L K L$, CD164 sialomucin-like-2 (CD164L2) protein, galanin receptor 3 (GAlR3), 5'-nucleotidase cytosolic II (NT5C2), elongator acetyltransferase complex subunit 5 (ELP5) and afamin $(A F M)$. The CD164L2 protein has been associated with essential hypertension in a recent study (23) and was found to be highly overexpressed in our results. GAlR3, the activation of which promotes adult neural stem cell survival in response to a diabetic milieu (24) was also highly overexpressed. Of note, the $M L K L$ gene transcripts were 8-fold higher in the SPARC-overexpressing cells, the $M L K L$ gene has a key role in a programmed cell death known as necroptosis. Of some importance in the AML transformed from the MDS cell model system, may be the underexpression of NT5C2, which has been previously involved in the relapse of acute lymphoblastic leukemia in patients (25). In the present study, the NT5C2 expression levels were decreased in SPARC-overexpressing SKM-1 cells following Ara-C treatment. ELP5 was found to have significantly less transcripts in SPARC-overexpressing cells. ELP5 was initially identified as a component of a hyperphosphorylated RNA polymerase II (RNAPII) holoenzyme isolated from budding yeast chromatin and subsequently from human melanoma cells (26). AFM, one of the newest members of albumins, is considered a good prognosis marker in ovarian cancer (27-29). The mRNA expression levels of these genes were tested by qPCR to validate our microarray results. As shown in Fig. 5A and B, the RT-PCR results were in agreement with the alterations in gene expression, as was evident from the microarray experiment results, for all six genes.

\section{Discussion}

There is abundant evidence of the role of SPARC in hematological and other malignancies, however, this is complex and differential according to the cell type and the malignancy entity $(16,18,30)$. Upregulation of the SPARC gene expression levels has been associated with the immunotherapeutic agent, lenalidomide, when treating patients with MDS characterized 
A
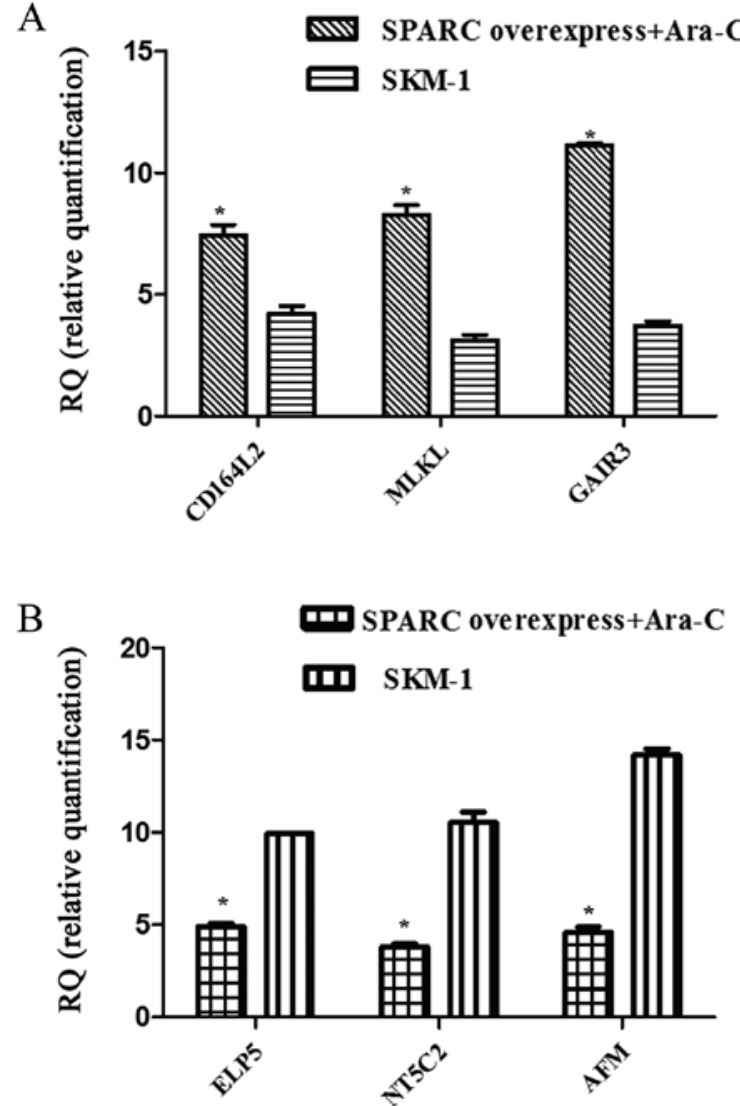

Figure 5. Validating microarray data by qPCR. (A) CD164L2, MLKL and GAlR3 were increased when SPARC overexpression was associated with Ara-C. The $2^{-\triangle \Delta C T}$ value increased 6.81-14.37 vs. other groups. (B) ELP5, NT5C2 and AFM expression was decreased to 3.67-13.55. SPARC, secreted protein acidic and rich in cysteine; CD164L2, CD164 silomucin-like-2; MLKL, mixed lineage kinase domain-like; GALR3, galanin receptor 3; ELP5, elongator acetyltransferase complex subunit 5; NT5C2, 5'-nucleotidase cytosolic II; AFM, afamin; Ara-C, cytosine arabinoside.

by the chromosomal deletion in the $5 \mathrm{q}$-segment (30). Further association of SPARC expression to MDS treatment management has yet to be established. In a previous study (22), we developed a lentiviral vector carrying a short hairpin RNA silencer for the $S P A R C$ gene and we employed this RNA interfering technology to knock down SPARC expression in SKM-1 cells. SPARC knockdown inhibited the proliferation of SKM-1 cells by inducing cell cycle arrest at the G1/G0 phase and apoptosis through p53 elevation of caspase- 9 and -3 and Fas at the mRNA and protein levels.

In the course of this present study, we constructed a SPARC-expressing lentiviral vector (pGC-GV-SPARC) and used it to stably transduce the MDS/AML SKM-1 cell line, in order to achieve ectopic overexpression of the SPARC protein. The SKM-1 cell line that was selected for infection is an MDS transformed to AML cell line, characterized by karyotypic pathological findings including del(9q), i(17q) and $t(17 p)$, but not carrying a $5 \mathrm{q}$-deletion. Following transduction by infection of the pGC-GV-SPARC vector into the SKM-1 cell line, $>60 \%$ of the cells were found to ectopically stably express the GFP protein proving the correct function of the construct and the promoter of the vector.

Upregulation of the SPARC mRNA levels was verified by qPCR against the normal SKM-1 SPARC mRNA levels and with those of the infected cells with an empty negative control vector. Overexpression of the SPARC protein was detected and confirmed by western blot analysis. The presence of increased SPARC protein in the cells was repeatedly associated with a recorded inhibition of the proliferation of the SKM-1 cells transduced by the pGC-GV-SPARC vector, in comparison to normal SKM-1 cells and SKM-1 cells infected with an empty vector. The results from the MTS assay that was used for measuring the proliferation rates, suggested a possible increase in the apoptosis of the pGC-GV-SPARC-infected cells. Further assessment of the apoptotic status of the pGC-GV-SPARC-infected cells by flow cytometry and microscopy demonstrated a significant increase in the number of pro-apoptotic and dead cells of the transduced cell line, their numbers reaching $30 \%$ of the cells tested. This result paralleled the observed inhibition of the proliferation effect that SPARC overexpression exerted on the cells. Empty vector-infected cells that were used as a negative control demonstrated minimal or no increase in apoptosis of the cells, thus excluding the factor of lentiviral vector infection as the causing agent of death. Of note, these results differ from the recent findings in AML patients and cells where it has been shown that an increased level of SPARC expression either ectopically in vitro, or when detected in ex vivo primary leukemic blasts from AML patients, contributes to leukemia growth in vitro and aggressive disease in vivo (18). By contrast, the results from this and a previous study (22), exhibit proliferation inhibition and increased cell death after SPARC overexpression or silencing in the AML/MDS SKM-1 cell line. These contradicting results of SPARC involvement in the biology of different cell types, underscores the pleiotropic function and effects of SPARC protein in the cell life cycle and its complex role in disease where its involvement is unique sometimes promoting growth and survival, whereas it may also lead to inhibition of proliferation and apoptosis.

Having established that proliferation was inhibited and apoptosis was promoted in the AML/MDS SPARC-overexpressing cell model, we assessed the effects of treatment of the cells with Ara-C, a chemotherapeutic agent used in the clinical management of high-risk MDS patients. Notable finding from our control samples was the small or absence of difference between the apoptotic effect of SPARC overexpression in the cells alone, and the treatment of normal SKM-1 cells with Ara-C. The two groups exhibited increased cell apoptosis close to $20-30 \%$ of the cells, demonstrating a similar apoptotic effect from the treatment of the normal SKM-1 cells with Ara-C and that of SPARC overexpression. Results of the flow cytometric analysis showed that apoptosis of SPARC-overexpressing cells following treatment with Ara-C increased 50\% from the non-treated controls and 30\% from the normal SKM-1 cells when treated with Ara-C. These conditions, suggested a synergy of the mechanisms leading to cell death or possibly a sensitization effect of one of the two conditions to the other.

In our microarray comparison study between SKM-1 cells treated with Ara-C and SKM-1 cells overexpressing SPARC and treated with Ara-C, we identified that the $M L K L$ gene was extensively upregulated in the SPARC-overexpressing cells when treated with Ara-C. MLKL is a major component of the TNF-induced suicidal signaling pathway known 
as necroptosis (31). This is a condition of programmed cell death that is characterized by an increase in intracellular $\mathrm{Ca}^{2+}$, generation of reactive oxygen species (ROS), intracellular acidity, depletion of ATP, and, eventually, plasma membrane rupture $(31,32)$. The process of necroptosis remains largely elusive, but it is known to involve the formation of the 'necrosome', a multiprotein complex mainly comprising the proteins RIPK1, RIPK-3 and MLKL. Following the phosphorylation of MLKL from RIPK3 (32), while part of the necrosome, MLKL translocates by means of lipid rafts to the plasma membrane and triggers the influx of $\mathrm{Ca}^{2+}$ through TRPM7 ion channels, a process that disrupts the integrity of the plasma membrane, ultimately leading to cell death (33). In the microarray results, we found that $M L K L$ gene expression was upregulated $>8$ times in the SPARC-overexpressing cells treated with Ara-C compared to the normal SKM-1 cells treated with Ara-C. It is therefore possible that SPARC overexpression from cells treated with Ara-C drives the cells to the process of necroptosis. Considering that SPARC is a secreted protein, it remains unclear whether such an effect may be exerted intracellularly to the SPARC-overexpressing cells or after secreted SPARC protein acts on targeted cells, effectively activating the TNF-induced necroptosis to these cells. This programmed cell death has been involved in hematological malignancies, when chronic lymphocytic leukemia cells were reported to have defects in components involved in the regulation of necroptosis such as RIP3 and the deubiquitination of cylindromatosis (CYLD), an enzyme directly regulating RIP1 ubiquitination (34). Examination of our microarray data for the behavior of the remaining known necroptosis pathway players, those of RIP1, RIP3, and caspase-8, showed no difference in the expression levels as compared to the normal levels. Another noteworthy finding was the significant decrease in NT5C2 gene expression. This gene encodes for a hydrolase that is involved in the cellular purine metabolism by acting primarily on inosine 5'-monophosphate and other purine nucleotides. A direct relationship was demonstrated between acquired somatic mutations and chemoresistance to a specific class of drugs used in treatment, those of the purine analogues 6-MP and 6-TG, but not across other cytotoxic agents including Ara-C. Previous results have shown that NT5C2 expressed in blast cells predicts survival in acute myeloid leukemia patients treated with cytarabine, suggesting NT5C2 is involved in Ara-C metabolism (35).

In conclusion, we present evidence that SPARC overexpression drives SKM-1 cells to the inhibition of proliferation and apoptosis, a condition that was also achieved in our previous study by silencing SPARC expression in the same cells. Treatment of SPARC-overexpressing SKM-1 cells with Ara- $\mathrm{C}$ results in an additive or even synergistic effect on the programmed cell death leading to a substantially increased number of dying cells, compared to what is achieved with each condition alone (SPARC overexpression or Ara-C treatment). The process may involve an alternative pathway of cell death other than apoptosis, such as the necroptosis pathway of programmed cell death, mediated by the protein MLKL which was found to be highly overexpressed in our microarray results. In combination with the results from a previous study, these results suggest that SPARC protein has a complex role in cellular biology, acting in alternative pathways of programmed cell death depending on the stringency of the stress conditions of the cellular environment. It becomes evident that the lack and overexpression of the SPARC gene are two conditions driving the AML/MDS SKM-1 cells to programmed cell death, possibly through alternative signaling pathways.

\section{Acknowledgements}

This study was supported by the National Natural Science Foundation of China (no. 30971277 and 81250034), the Natural Science Foundation of Chongqing (CSTC, no. 2009BB5070), the Health Bureau of Chongqing (2013-2-023) and the Project Foundation of Chongqing Municipal Education Committee (2013).

\section{References}

1. Heaney ML and Golde DW: Myelodysplasia. N Engl J Med 340: 1649-1660, 1999.

2. Malcovati L, Hellström-Lindberg E, Bowen D, Adès L, Cermak J, Del Cañizo C, Della Porta MG, Fenaux P, Gattermann N, Germing U, et al; European Leukemia Net: Diagnosis and treatment of primary myelodysplastic syndromes in adults: recommendations from the European LeukemiaNet. Blood 122: 2943-2964, 2013

3. Nimer SD: Myelodysplastic syndromes. Blood 111: 4841-4851, 2008.

4. Davids MS and Steensma DP: The molecular pathogenesis of myelodysplastic syndromes. Cancer Biol Ther 10: 309-319, 2010.

5. Jädersten $M$ and Hellström-Lindberg E: New clues to the molecular pathogenesis of myelodysplastic syndromes. Exp Cell Res 316: 1390-1396, 2010.

6. Boultwood J, Lewis S and Wainscoat JS: The 5q-syndrome. Blood 84: 3253-3260, 1994.

7. Mauritzson N, Albin M, Rylander L, Billström R, Ahlgren T, Mikoczy Z, Björk J, Strömberg U, Nilsson PG, Mitelman F, et al: Pooled analysis of clinical and cytogenetic features in treatment-related and de novo adult acute myeloid leukemia and myelodysplastic syndromes based on a consecutive series of 761 patients analyzed 1976-1993 and on 5098 unselected cases reported in the literature 1974-2001. Leukemia 16: 2366-2378, 2002.

8. Giagounidis AA, Germing U and Aul C: Biological and prognostic significance of chromosome $5 \mathrm{q}$ deletions in myeloid malignancies. Clin Cancer Res 12: 5-10, 2006.

9. Wang L, Fidler C, Nadig N, Giagounidis A, Della Porta MG, Malcovati L, Killick S, Gattermann N, Aul C, Boultwood J, et al: Genome-wide analysis of copy number changes and loss of heterozygosity in myelodysplastic syndrome with del(5q) using high-density single nucleotide polymorphism arrays. Haematologica 93: 994-1000, 2008.

10. Giagounidis AA, Germing U, Wainscoat JS, Boultwood J and Aul C: The 5q- syndrome. Hematology 9: 271-277, 2004.

11. Rotllant J, Liu D, Yan YL, Postlethwait JH, Westerfield M and Du SJ: Sparc (osteonectin) functions in morphogenesis of the pharyngeal skeleton and inner ear. Matrix Biol 27: 561-572, 2008.

12. Swaminathan SS, Oh DJ, Kang MH, Ren R, Jin R, Gong H and Rhee DJ: Secreted protein acidic and rich in cysteine (SPARC)-null mice exhibit more uniform outflow. Invest Ophthalmol Vis Sci 54: 2035-2047, 2013.

13. Hsiao YH, Lien HC, Hwa HL, Kuo WH, Chang KJ and Hsieh FJ: SPARC (osteonectin) in breast tumors of different histologic types and its role in the outcome of invasive ductal carcinoma. Breast J 16: 305-308, 2010.

14. Wiese AH, Auer J, Lassmann S, Nährig J, Rosenberg R, Höfler H, Rüger $\mathrm{R}$ and Werner M: Identification of gene signatures for invasive colorectal tumor cells. Cancer Detect Prev 31: 282-295, 2007.

15. DiMartino JF, Lacayo NJ, Varadi M, Li L, Saraiya C, Ravindranath Y, Yu R, Sikic BI, Raimondi SC and Dahl GV: Low or absent SPARC expression in acute myeloid leukemia with MLL rearrangements is associated with sensitivity to growth inhibition by exogenous SPARC protein. Leukemia 20: 426-432, 2006. 
16. Giagounidis A, Mufti GJ, Fenaux P, Germing U, List A and MacBeth KJ: Lenalidomide as a disease-modifying agent in patients with del $(5 q)$ myelodysplastic syndromes: linking mechanism of action to clinical outcomes. Ann Hematol 93: 1-11, 2014.

17. Giallongo C, La Cava P, Tibullo D, Barbagallo I, Parrinello N, Cupri A, Stagno F, Consoli C, Chiarenza A, Palumbo GA, et al: SPARC expression in CML is associated to imatinib treatment and to inhibition of leukemia cell proliferation. BMC Cancer 13: 60, 2013.

18. Alachkar H, Santhanam R, Maharry K, Metzeler KH, Huang X, Kohlschmidt J, Mendler JH, Benito JM, Hickey C, Neviani P, et al: SPARC promotes leukemic cell growth and predicts acute myeloid leukemia outcome. J Clin Invest 124: 1512-1524, 2014

19. Said N, Frierson HF Jr, Chernauskas D, Conaway M, Motamed K and Theodorescu D: The role of SPARC in the TRAMP model of prostate carcinogenesis and progression. Oncogene 28: 3487-3498, 2009.

20. de Vries JF, Falkenburg JH, Willemze R and Barge RM: The mechanisms of Ara-C-induced apoptosis of resting B-chronic lymphocytic leukemia cells. Haematologica 91: 912-919, 2006.

21. Germing U,Kobbe G, Haas R and Gattermann N: Myelodysplastic syndromes: diagnosis, prognosis, and treatment. Dtsch Arztebl Int 110: 783-790, 2013.

22. Nian Q, Xiao Q, Wang L, Luo J, Chen LP, Yang ZS and Liu L: SPARC silencing inhibits the growth of acute myeloid leukemia transformed from myelodysplastic syndrome via induction of cell cycle arrest and apoptosis. Int J Mol Med 33: 856-862, 2014

23. Lu J, Li M, Zhang R, Hu C, Wang C, Jiang F, Yu W, Qin W, Tang S and Jia W: A common genetic variant of FCN3/CD164L2 is associated with essential hypertension in a Chinese population. Clin Exp Hypertens 34: 377-382, 2012.

24. Mansouri S, Barde S, Ortsäter H, Eweida M, Darsalia V, Langel U, Sjöholm A, Hökfelt T and Patrone C: GalR3 activation promotes adult neural stem cell survival in response to a diabetic milieu. J Neurochem 127: 209-220, 2013.

25. Meyer JA, Wang J, Hogan LE, Yang JJ, Dandekar S, Patel JP, Tang Z, Zumbo P, Li S, Zavadil J, et al: Relapse-specific mutations in NT5C2 in childhood acute lymphoblastic leukemia. Nat Genet 45: 290-294, 2013.

26. Close P, Gillard M, Ladang A, Jiang Z, Papuga J, Hawkes N, Nguyen L, Chapelle JP, Bouillenne F, Svejstrup J, et al: DERP6 (ELP5) and C3ORF75 (ELP6) regulate tumorigenicity and migration of melanoma cells as subunits of elongator. J Biol Chem 287: 32535-32545, 2012.
27. Dieplinger B, Egger M, Gabriel C, Poelz W, Morandell E, Seeber B, Kronenberg F, Haltmayer M, Mueller T and Dieplinger H: Analytical characterization and clinical evaluation of an enzymelinked immunosorbent assay for measurement of afamin in human plasma. Clin Chim Acta 425: 236-241, 2013.

28. Jackson D, Craven RA, Hutson RC, Graze I, Lueth P, Tonge RP, Hartley JL, Nickson JA, Rayner SJ, Johnston C, et al: Proteomic profiling identifies afamin as a potential biomarker for ovarian cancer. Clin Cancer Res 13: 7370-7379, 2007.

29. Melmer A, Fineder L, Lamina C, Kollerits B, Dieplinger B, Braicu I, Sehouli J, Cadron I, Vergote I, Mahner S, et al: Plasma concentrations of the vitamin E-binding protein afamin are associated with overall and progression-free survival and platinum sensitivity in serous ovarian cancer - a study by the OVCAD consortium. Gynecol Oncol 128: 38-43, 2013.

30. Pellagatti A, Jädersten M,Forsblom AM,Cattan H, Christensson B, Emanuelsson EK, Merup M, Nilsson L, Samuelsson J, Sander B, et al: Lenalidomide inhibits the malignant clone and up-regulates the SPARC gene mapping to the commonly deleted region in 5q-syndrome patients. Proc Natl Acad Sci USA 104: 11406-11411, 2007.

31. Giampietri C, Starace D, Petrungaro S, Filippini A and Ziparo E: Necroptosis: molecular signalling and translational implications. Int J Cell Biol 2014: 490275, 2014

32. Murphy JM, Czabotar PE, Hildebrand JM, Lucet IS, Zhang JG, Alvarez-Diaz S, Lewis R, Lalaoui N, Metcalf D, Webb AI, et al: The pseudokinase MLKL mediates necroptosis via a molecular switch mechanism. Immunity 39: 443-453, 2013.

33. Cai Z, Jitkaew S, Zhao J, Chiang HC, Choksi S, Liu J, Ward Y, Wu LG and Liu ZG: Plasma membrane translocation of trimerized MLKL protein is required for TNF-induced necroptosis. Nat Cell Biol 16: 55-65, 2014.

34. Moquin DM, McQuade T and Chan FK: CYLD deubiquitinates $\mathrm{RIP1}$ in the $\mathrm{TNF} \alpha$-induced necrosome to facilitate kinase activation and programmed necrosis. PLoS One 8: e76841, 2013.

35. Galmarini CM, Thomas X, Graham K, El Jafaari A, Cros E, Jordheim L, Mackey JR and Dumontet C: Deoxycytidine kinase and $\mathrm{cN}$-II nucleotidase expression in blast cells predict survival in acute myeloid leukaemia patients treated with cytarabine. Br J Haematol 122: 53-60, 2003 